\title{
Control of Multi-Terminal VSC-HVDC System to Integrate Large Offshore Wind Farms
}

\author{
Xiongguang Zhao, Qiang Song, Hong Rao, Xiaoqian Li, Xiaolin Li, and Wenhua Liu
}

\begin{abstract}
This paper presents operation and control strategies of multi-terminal HVDC transmission system (MTDC) using voltage source converters (VSCs) for integrating large offshore wind farms. The framework and operation principles of the proposed system are described and control strategies for coordinating various VSCs are proposed. DC voltage control based on the DC voltage-current (V-I) droop characteristic of grid side converters is implemented, to ensure stable system operation and flexible power dispatch between various onshore $\mathrm{AC}$ grids. To validate the performance of the proposed control strategies, a typical four terminal MTDC networks, which connecting two offshore wind farms with two onshore AC grids, is established in PSCAD/EMTEC. Simulation results under normal and abnormal operation conditions verify the satisfactory performance of the proposed control strategy and accuracy of the theoretical analysis.
\end{abstract}

Index Terms-Control, HVDC, multi-terminal, voltage source converter, wind farm.

\section{INTRODUCTION}

As wind power is a kind of environmental friendly energy and abundantly available in nature, the China government has set a target of developing $200 \mathrm{GW}$ wind farms by 2020 in order to deal with global warming and achieve a goal that $15 \%$ of power consumption is provided by renewable energy. Offshore wind farms will increase to $30 \mathrm{GW}$ according to the target and are developing rapidly in recent years. Integrating the offshore wind farms to the grid over a long distance is one of the main challenges facing researchers. Previous studies have indicated that high voltage DC (HVDC) transmission has a lot of advantages over traditional AC transmission, including fewer cables required, not affected by the cable charging current and flexibly controlled power flow [1], [2].Compared with line commutated converter (LCC) HVDC, the VSC-HVDC shows many advantages [3]-[7]. These include avoiding commutation failure, the independent control of active and reactive power, no voltage polarity reversal required to reverse power, producing less harmonic, less filters required and continuous $\mathrm{AC}$ bus voltage regulation. Because of the above reasons, VSC-HVDC is considered as a promising solution to integrating large offshore wind farms into onshore AC grids and has attracted a lot of research [5]-[8].

In VSC-HVDC, VSC multi-terminal HVDC (VSC-MTDC)

Manuscript received October 15, 2012; revised November 22, 2012.

Xiongguang Zhao, Qiang Song, Xiaoqian Li, and Wenhua Liu are with the Department of Electrical Engineering, Tsinghua University, Beijing, 10084, China (e-mail: zhaoxg07@ sina.com)

Hong Rao and Xiaolin Li are with CSG Technology Research Center,Guangzhou,Guangdong, 510623,China (e-mail: raohong@csg.cn) transmission system, which consists of more than two converters connected through DC cables, can reduce the number of converters and improve the flexibility and reliability, when compared to numerous point to point HVDC systems. But the challenge is that the operation and control of VSC-MTDC is more complex. Various control strategies have been proposed for VSC-MTDC [9]-[11]. In [9], a voltage margin control method was proposed, in which each converter station in the system was given a marginally offset DC voltage reference. At any time, only one converter is used to control the DC voltage in this method. Reference [10] designed a control method based on the voltage-power characteristic of the converters for a MTDC system without fast communication. In [11], a current matching control was used to control the DC current and power sharing ratio among the AC girds. This kind of control depended on the communication equipment to transmit current information. The deficiency of the above control methods is that they can't allow multiple converters to control the DC voltage and change the power sharing ratio between the receiving $\mathrm{AC}$ girds without communications simultaneously.

This paper proposes a control method, which allows multiple converters to control the DC voltage and can dispatch the power between the receiving AC girds of MTDC system at a pre-defined ratio without the use of communications between terminals. The control method combines the voltage control with the V-I characteristic of gird side VSCs (GSVSCs). The paper is organized as follows. Section $I I$ introduces the system configuration and describes the main control strategies. Simulation results under both normal and abnormal conditions are given in section III to validate the performance of the proposed control strategies. Finally section IV draws the conclusions.

\section{CONTROL Strategy}

\section{A. Control Target of the VSC-MTDC System}

As shown in Fig.1, a typical configuration, which consists of two wind farm VSCs (WFVSCs) and two GSVSCs, is used to investigate the MTDC system. It is easy to extend it to the systems with more terminals according to similar principles. The wind turbines in the two offshore wind farms are considered to be connected together by local AC networks. The two WFVSCs convert AC to DC and then the DC cables transmit the total connected power to the onshore GSVSCs. The two GSVSCs then convert the de voltage to ac voltage and transmit the power to the AC girds. The two WFVSCs implement frequency and AC voltage control at the point of common connection (PCC) with wind farms. Each 
GSVSC is equipped with an inner current controller and an outer DC voltage regulator. In addition they can also control the AC voltage or reactive power flow.

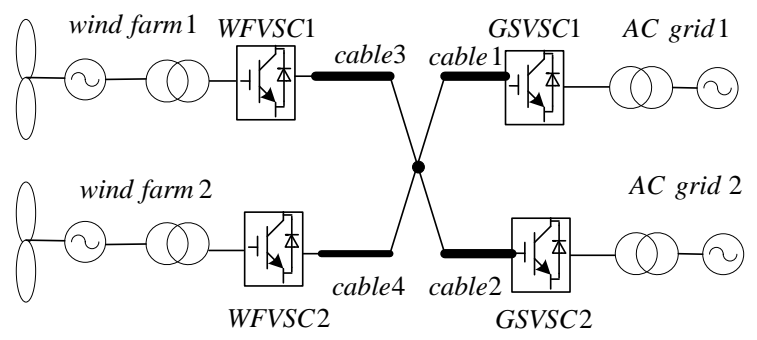

Fig. 1. A four terminal MTDC system

Under normal operation, the DC voltage of MTDC system must be maintained at a set value within a limited range. Abnormal DC voltage can disrupt the normal operation and even cause the trip of the system. A stable DC voltage indicates that the target of power balancing has been achieved among the multi-terminals. Therefore, the two WFVSCs are controlled to provide a constant AC voltage for the PCCs with wind farms. The control target of the two GSVSCs are designed to control the DC voltage to ensure the power generated by the offshore wind farms can be transmitted to the two AC girds and to dispatch the power between the two AC girds at a set ratio. Since the control of the WFVSCs is simple and clear, this paper focuses on the control of the two GSVSCs.

\section{B. V-I Droop Characteristics}

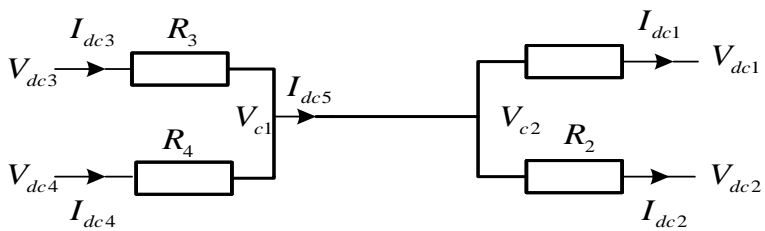

Fig. 2. DC equivalent circuit for the MTDC

Fig. 2 shows the steady state DC equivalent circuit of the MTDC system shown in Fig. 1. $R_{1} \sim R_{4}$ represent the equivalent DC resistances of DC cable1 cable4, respectively. $V_{d c 1} \sim V_{d c 4}$ and $I_{d c 1} \sim I_{d c 4}$ are the responding DC voltages and currents of the four VSCs. For GSVSC1 and GSVSC2, the relationship between the $\mathrm{DC}$ voltages are given by:

$$
V_{d c 1}=V_{c 2}-I_{d c 1} R_{1} \quad V_{d c 2}=V_{c 2}-I_{d c 2} R_{2}
$$

where $V_{c 2}$ is the voltage of the common point as shown in Fig. 2. According to (1), following equation can be obtained:

$$
V_{d c 2}=V_{d c 1}+I_{d c 1} R_{1}-I_{d c 2} R_{2}
$$

The control method proposed in this paper is based on the DC V-I droop characteristics of the two GSVSCs, as shown in Fig. 3. According to Fig.3, the DC voltage can be obtained by:

$$
V_{d c 1}=V_{\text {min }}+k_{1} I_{d c 1} \quad V_{d c 2}=V_{\text {min }}+k_{2} I_{d c 2}
$$

where $V_{\min }$ is the minimum DC voltage set by the system operator and $k_{1}$ and $k_{2}$ are the slops of the V-I characteristics of GSVSC1 and GSVSC2, respectively. The control strategies are designed to dispatch the power between the two
GSVSCs (AC girds) at a set ratio represented by $n$. Because the DC voltages are approximately equal, the power sharing ratio can be expressed as:

$$
\frac{P_{1}}{P_{2}}=n=\frac{I_{d c 1} V_{d c 1}}{I_{d c 2} V_{d c 2}} \approx \frac{I_{d c 1}}{I_{d c 2}}
$$

Substituting (3) into (2):

$$
V_{d c 2}-V_{d c 1}=k_{2} I_{d c 2}-k_{1} I_{d c 1}=I_{d c 1} R_{1}-I_{d c 2} R_{2}
$$

Then substituting (5) into (4):

$$
\frac{I_{d c 1}}{I_{d c 2}}=\frac{R_{2}+k_{2}}{R_{1}+k_{1}}=n
$$

That is:

$$
k_{2}=n k_{1}+n R_{1}-R_{2}
$$

Equation (7) indicates that if the value of $R_{1}$ and $R_{2}$ are known, the value of $k_{1}$ and $k_{2}$ can be adjusted to get the required power sharing ratio $n$.

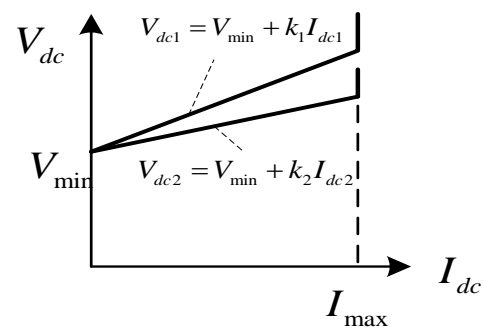

Fig. 3. V-I droop characteristics of GSVSCs

\section{Control Strategies of GSVSCS}

As the operation and control of VSC-HVDC have been well studied in [8]-[9], this paper focuses on the DC voltage control and the principle of power dispatch of MTDC system. Fig.4 shows the simplified control systems of each GSVSC. A proportional controller is used instead of the usual PI controller to control the DC voltage because there will be a difference between the DC voltage, $V_{d c}$, and its reference value, $V_{d c r e f}$. $V_{d c}$ is designed to change based on the V-I droop characteristic of each GSVSC and $V_{d c r e f}$ is set as the minimum DC voltage which is corresponding to $V_{\min }$ in (3). The control outputs, $i_{d}{ }^{*}$ and $i_{q}{ }^{*}$, are the reference value for the $d$-axis and $q$-axis component of the gird current respectively, which are associated with the active and reactive power. The inner current control loop is designed to regulate $i_{d}$ and $i_{q}$ to track $i_{d}{ }^{*}$ and $i_{q}{ }^{*}$ respectively and produce the $d$-axis and $q$-axis reference voltage, $V_{d r e f}$ and $V_{\text {qref }}$, for controlling the GSVSCs.

The proportional gain is $k_{p}$. Based on the control systems as shown in Fig. 4, the relationship between $V_{d c}$ and $V_{d c r e f}$ can be obtained in order to associate them with the $V-I$ droop characteristics of GSVSCs and the relationship can be expressed as:

$$
V_{d c r e f}-V_{d c}=-\frac{i_{d}^{*}}{k_{p}}=-\frac{i_{d}}{k_{p}}=-\frac{i_{d}}{I_{d c}} * \frac{I_{d c}}{k_{p}}
$$

In the rotating reference frame, the $d$-axis voltage $V_{\mathrm{d}}$ is 
aligned with the phase voltage $V_{a}$, and the $q$-axis voltage $V_{q}$ is zero. When the quadrature Park transformation is used, ignoring the power loss, the power balance equation of each GSVSC can be expressed as:

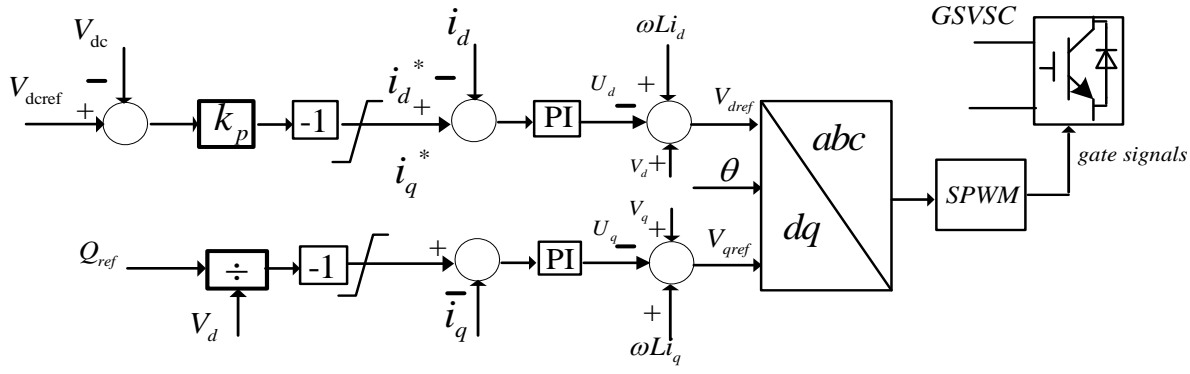

Fig. 4. Control systems of each GSVSC

$$
P=V_{d c} I_{d c}=V_{d} i_{d}
$$

For the pulse width modulation (PWM) control, the modulation index is:

$$
M=\frac{\sqrt{2} V_{a}}{V_{d c} / 2}
$$

Thus, the ratio of $I_{d c}$ and $i_{d}$ is given by:

$$
\frac{I_{d c}}{i_{d}}=\frac{V_{d}}{V_{d c}}=\frac{\sqrt{3 / 2} * \sqrt{2} V_{a}}{V_{d c}}=\frac{\sqrt{6}}{4} * \frac{\sqrt{2} V_{a}}{V_{d c} / 2}=\frac{\sqrt{6}}{4} M=k_{c}
$$

In (11), $k_{c}$ is a constant value which is proportional to $M$. Substituting (11) into (8):

$$
V_{d c r e f}-V_{d c}=-\frac{1}{k_{c}} * \frac{I_{d c}}{k_{p}}
$$

Assuming $K=-1 / k_{c} k_{p}$, equation (12) can be expressed as:

$$
V_{d c}=V_{d c r e f}+K I_{d c}
$$

This is the actual $V-I$ characteristic of each GSVSC. Comparing (13) with (3), it is obvious that $V_{d c r e f}$ is corresponding to $V_{\min }$ and $K$ is corresponding to slope $k_{1}$ or $k_{2}$. Thus, according to (7) and (13), a conclusion can be drawn that by adjusting the proportional gain $k_{p}$ to regulate the value of $K$ of each GSVSC, the power sharing ratio $n$ can be controlled to the pre-set value. In summary, $n$ is determined by the proportional gain $k_{p}$ of each GSVSC.

\section{Simulation Results}

The four terminals VSC-HVDC system, shown in Fig.1, was simulated using PSCAD/EMTDC. There are two 200 MW wind farms connected to the VSC-MTDC network. The four VSCs employ the two-level configuration with a switching frequency of $3.2 \mathrm{kHz}$. Each converter is rated at $300 \mathrm{MW}$. The rated DC voltage is $300 \mathrm{kV}$. DC cables were used to connect the four terminals and each cable has the equivalent parameters of $R=0.2 \Omega, L=0.005 \mathrm{H}$ and $C=5 \mu \mathrm{F}$. The reference dc voltage, $V_{d c r e f}$, of each GSVSC is set to $0.95 \mathrm{pu}$ $(285 \mathrm{kV})$.

\section{A. Step Change of Wind Farms}

Step changes of the input wind farm power were used to evaluate the control strategies. The injected power from wind farm 1 was changed from $0.5 \mathrm{pu}$ to $1.0 \mathrm{pu}$ at $7 \mathrm{~s}$ and recovered to $1.0 \mathrm{pu}$ at $12 \mathrm{~s}$, while the injected power from wind farm 2 remained 1.0pu all the time. Regulate $k_{p}$ of each GSVSC to set

$k_{1}=k_{2}=30$. Thus the power sharing ratio $n=1$.Fig. 5 shows the simulation results.

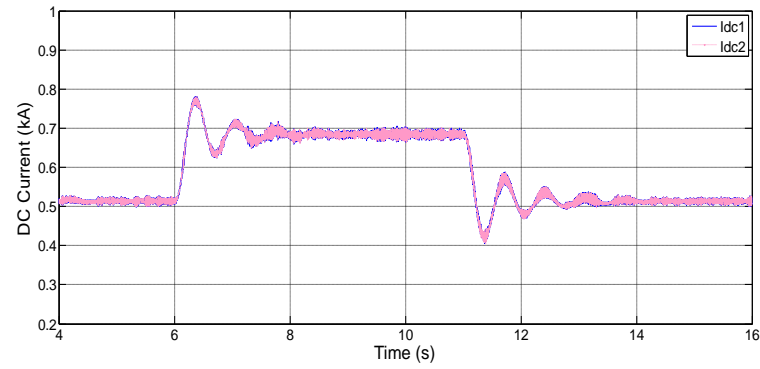

(a) DC current of GSVSCs

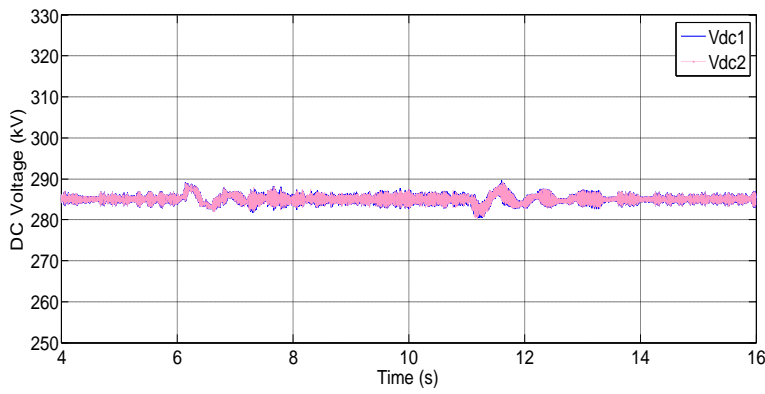

(b)DC voltage of GSVSCs

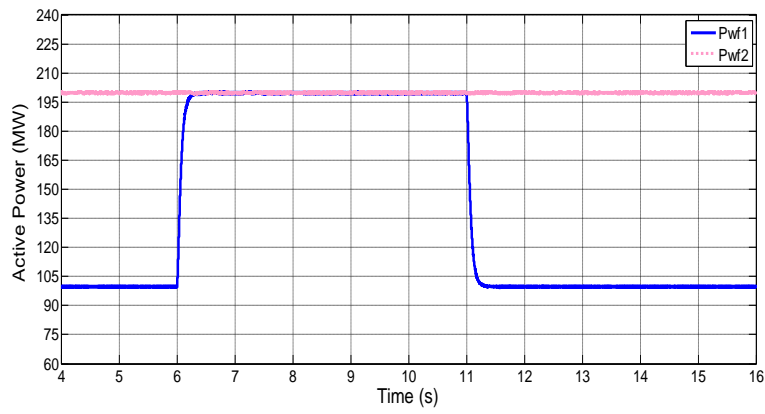

(c)Active power of wind farms 


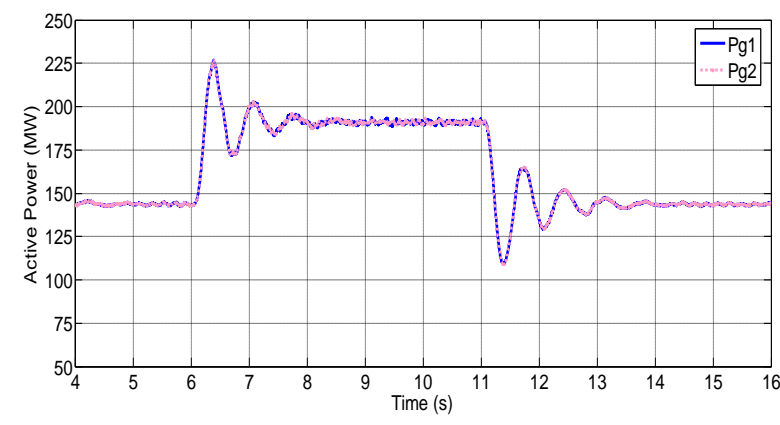

(d)Active power of AC girds

Fig. 5. Simulation results with variable wind power

As illustrated in Fig.5 (a) and (b), the DC currents of GSVSCs and the DC voltages changed with the wind power. The DC voltages were maintained at nearly a constant value when the system came back to steady state, which means the power balancing is achieved. The total injected wind power was shared equally by AC gird 1 and 2, both of which are 142.5 MW in the period of 0-6s and the period of 14-16s. The total shared power was slightly less than the injected power duo to the power loss caused by VSCs and DC cables. It can be seen that the MTDC system has a good dynamic response when the injected wind farm power is changed.

\section{B. Step Change of Power Sharing Ratio}

Step changes of power sharing ratio were used to validate the power dispatch theory deduced above and further evaluate the control strategies. Regulate $k_{p}$ of each GSVSC to set the value of power sharing ratio $n$ according to the previously explained theory. Wind farm 1 provided $1.0 \mathrm{pu}$ power and wind farm 2 provided $0.5 \mathrm{pu}$ power all the time. The power sharing ratio changed from $n=2$ to $n=1.5$ at $6 \mathrm{~s}$ and changed back to $n=2$ at $11 \mathrm{~s}$. Fig. 6 shows the simulation results.

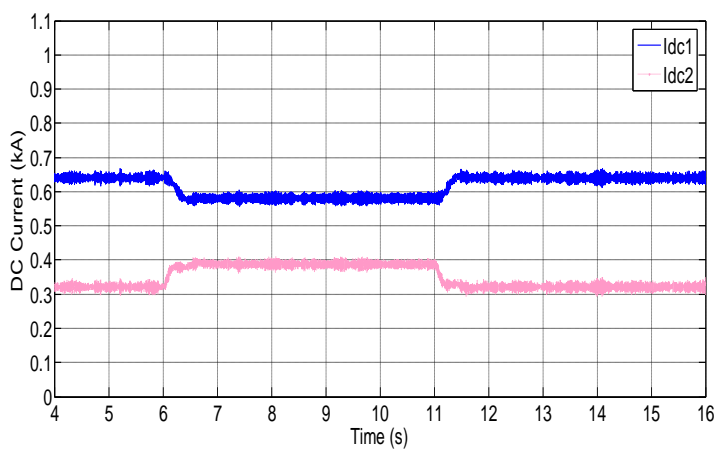

(a) DC current of GSVSCs

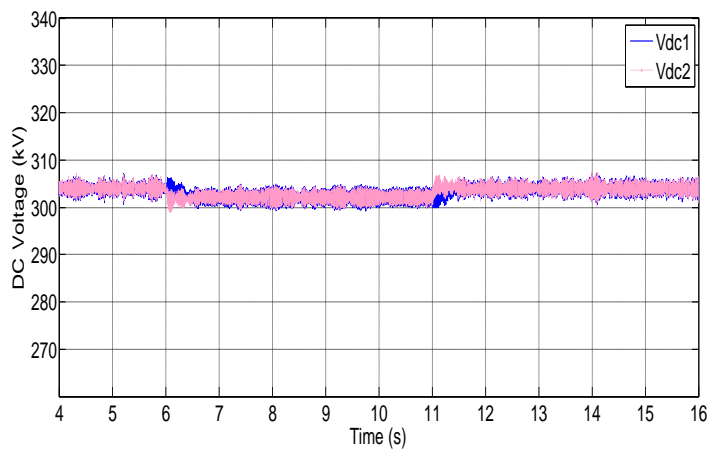

(b)DC voltage of GSVSCs

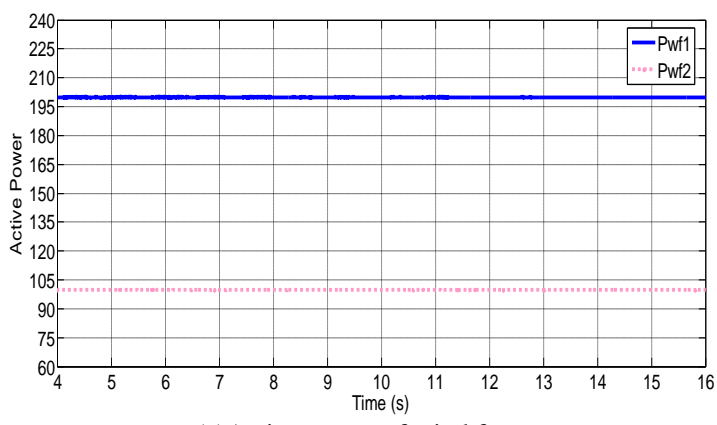

(c)Active power of wind farms

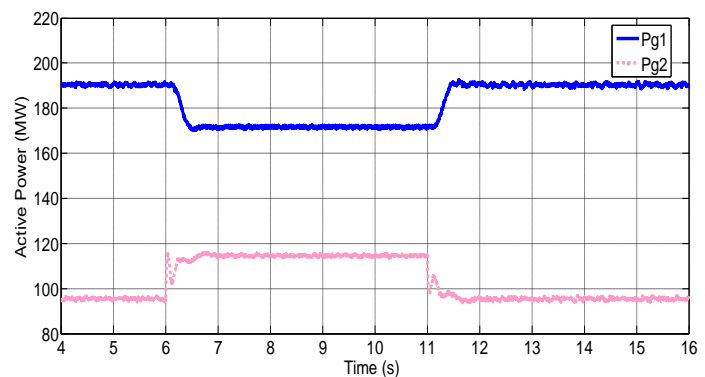

(d)Active power of AC girds

Fig. 6. Simulation results with variable power sharing ratio

As shown in Fig.6, the DC currents of GSVSCs varied with the change of the power sharing ratio. The DC voltages were almost unchanged. The total injected power was 300 MW. AC gird 1 and 2 absorbed 189.8 MW and 95.2 MW, respectively, between $0-6 \mathrm{~s}$ and 12-16s. The actual power sharing ratio was 1.994. While 171.3 MW and 114.7 MW were absorbed by AC gird 1 and 2, respectively, during 7-11s. The actual power sharing ratio was 1.493.Simulation results show that the actual power sharing ratio is almost identical with the theoretical value and the dynamic response is satisfactory.

\section{Simulation Results Under a Three Phase Fault}

A three phase to ground fault was applied at $4 \mathrm{~s}$ for duration of $0.2 \mathrm{~s}$ on the primary side of GSVSC2's coupling transformer. The injected power from wind farm 1 and wind farm 2 was $1.0 \mathrm{pu}$ and $0.5 \mathrm{pu}$, respectively. The power sharing ratio $n$ was set to 2 .

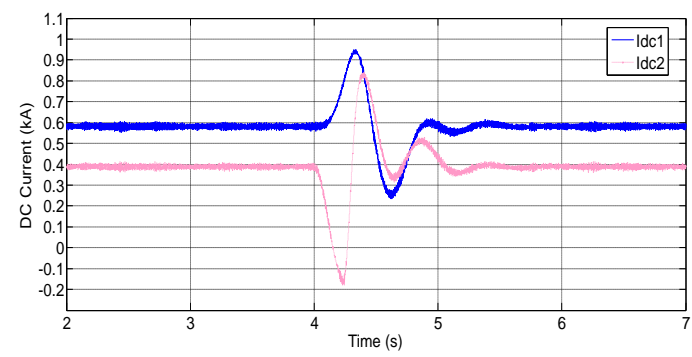

(a) DC current of GSVSCs

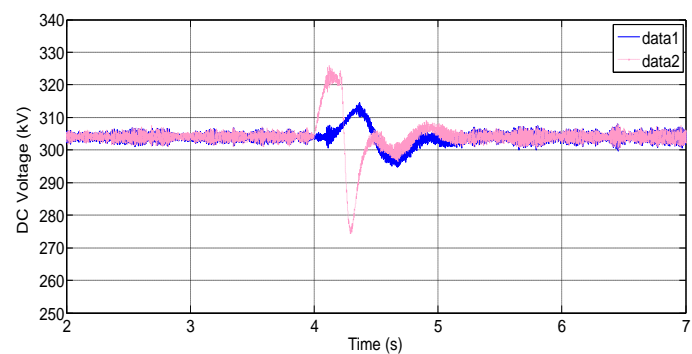

(b)DC voltage of GSVSCs 


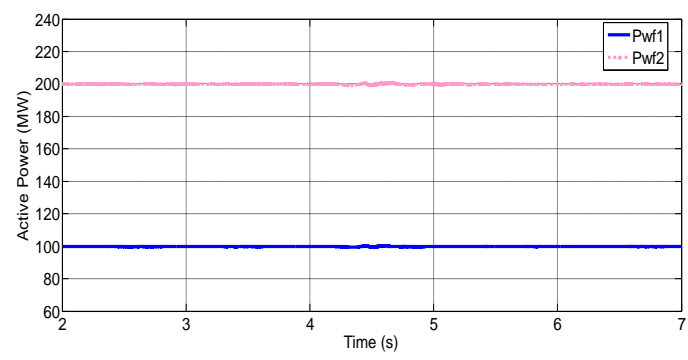

(c)Active power of wind farms

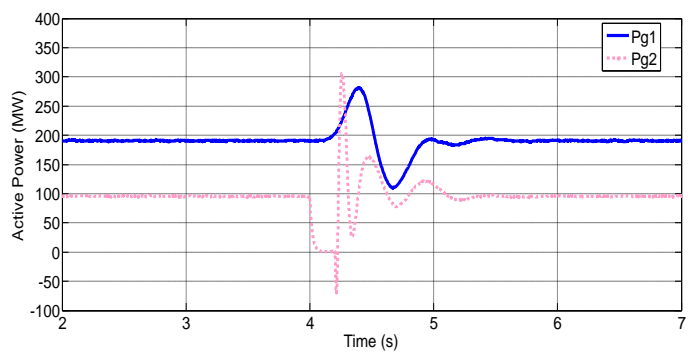

(d)Active power of AC girds

Fig. 7. Simulation results with a three phase fault

As shown in Fig. 7, before the fault was applied, AC grid 1 shared a power of $190 \mathrm{MW}$ and AC gird 2 shared a power of $96 \mathrm{MW}$. When the fault occurred at $4 \mathrm{~s}$, the power absorbed by AC gird 2 decreased to zero quickly and the surplus power caused by the power loss of GSVSC2 transferred to GSVSC1, thus the power absorbed by AC gird 1 increased. The DC voltages rose sharply. Especially the voltage of GSVSC2 rose to $325 \mathrm{kV}$ at $4.1 \mathrm{~s}$, which is $8.33 \%$ higher than the nominal voltage. The system recovered to normal operation within 1.5 second.

\section{CONCLUSIONS}

A typical four-terminal VSC-HVDC system was built for integrating large offshore wind farms. The operation principles and control strategies of the system were described. The two WFVSCs were controlled to establish a constant AC voltage and frequency for the PCCs with wind farms. DC voltage control based on the $V$-I characteristics of GSVSCs was designed to regulate DC voltage and coordinate power sharing between the two AC girds. The relationship between the power sharing ratio and proportional gain was described. The proposed control method allows multiple converters to control the DC voltage and dispatch the power at a set ratio in real time without fast communications. Simulations results under variable wind power and power sharing ratio have been presented to validate the performance of the proposed control strategies. The results show satisfactory dynamic response. The system can maintain stability and show good performance in a certain degree during large disturbance caused by three phase to ground fault on the AC gird.

\section{ACKNOWLEDGMENT}

The authors gratefully acknowledge the kind support of the National High Technology Research and Development of China 863 Program (NO.2011AA05A102).

\section{REFERENCES}

[1] N. M. Kirby, M. J. Luckett, L. Xu, and W. Siepmann, "HVDC Transmission for large off shore wind farms," IEE AC-DC Power Transmission, November 2001, London, no. 485, pp. 162-168.

[2] D. Jovcic, "Interconnecting offshore wind farms using multi-terminal VSC-based HVDC," IEEE PES General Meeting, 2006.

[3] A. Moharana, P. K. Dash, "Input-Output Linearization and Robust Sliding-Mode Controller for the VSC-HVDC Transmission Link," IEEE Transactions on Power Delivery, vol. 25, no. 3, 2010, pp. 1952-1961.

[4] R. Ottersten, J. Svensson, "Vector current controlled voltage source converter-deadbeat control and saturation strategies," Power Electronics, IEEE Transactions, vol. 17, no. 2, 2002, pp. 279-285.

[5] L. Bin, O. Boon-Teck, "Nonlinear Control of Voltage-Source Converter Systems," Power Electronics, IEEE Transactions, vol. 22, no. 4, 2007, pp. 1186-1195

[6] Z. G. Bin, X. Zheng, and W. G. Zhu, "Steady-state model and its nonlinear control of VSC-HVDC system," in Proc. of CSEE, vol. 1, 2002, pp. 17-22.

[7] N. Flourentzou, V. G. Agelidis, and G. D. Demetriades, "VSC-Based HVDC Power Transmission Systems: An Overview," IEEE Trans. Power Electronics, vol. 24, no. 3, March 2009, pp. 592-602.

[8] W. Lu and B. Ooi, "Optimal acquisition and aggregation of offshore wind power by multiterminal voltage-source HVDC," IEEE Transaction on Power Delivery, vol. 18, no. 1, pp. 201-206, 2003.

[9] T. Nakajima and S. A. Irokawa, "A control System for HVDC Transmission by Voltage Sourced Converters," IEEE Power Engineering Society Summer Meeting, Edmonton, pp. 1113-1119, 1999.

[10] G. P. Adam, S. J. Finny, B.W. Williams, and G. M. Burt, "Control of multi-terminal DC transmission system based on voltage source converters," in Proc. AC and DC Power Transmission, AC.DC. $9^{\text {th }}$ IET International Conference, 2010.

[11] J. Zhu, C. Booth, and G. P. Adam,"Current Matching Control system for Multi-Terminal DC transmission to integrate offshore wind farms," IET Renewable Power Generation, 2011.

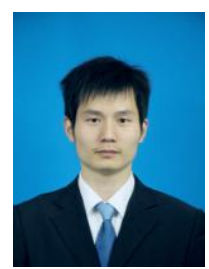

Xiongguang Zhao was born in Hubei, China, in August 1988. He received the BS Degrees from Tsinghua University, China in 2011, in Electrical Engineering. He is now a Master student in Department of Electrical Engineering of Tsinghua University, China. His main field of interest includes FACTS, and VSC-HVDC .

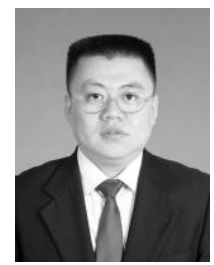

Qiang Song was born in Changchun, China in 1975. He received his B.E.E. degree and Ph.D degree from Tsinghua University, Beijing, China, both in Electrical Engineering, in 1998 and 2003 respectively. From 2003 to 2008 , he was a lecturer in the Department of Electrical Engineering at Tsinghua University, Beijing ,China. Since 2008, he has been an Associate Professor in the Department of Electrical Engineering at Tsinghua University. His main research interests are in high power electronic interfaces for utility system, Flexible AC Transmission System, VSC-HVDC system and custom power quality.

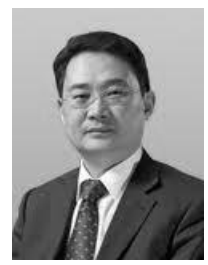

Hong Rao was born in Hubei, China in 1962. He received his B.E.E. degree from Huazhong University of Science and Technology, Wuhan, China, in 1983. He is currently an expert Engineer in Electric Power Research Institute of China Southern Power Grid. His main research interests are HVDC, VSC-HVDC, and power system analysis. 


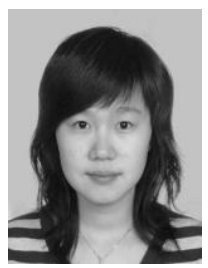

Xiaoqian Li was born in Hebei, China, in 1987. She received the B.S. degree in electrical engineering from North China Electrical Power University, Beijing, China, in 2010. She is now pursuing her Ph.D. degree in electrical engineering in Tsinghua University, Beijing, China. Her current research interests include high-power electronic, Flexible AC Transmission System and VSC-HVDC system.

Xiaolin Li was born in Hnan, China in 1963. He received his B.E.E degree from Wuhan University of Hydraulic and Electrical Engineering, China, in 1983. He received his M.E.E degree from Graduate School of North China Electric Power University, Beijing, China, in 1987. He is currently a senior Engineer in Electric Power Research Institute of China Southern Power Grid. His main research interests are HVDC, VSC-HVDC technology.

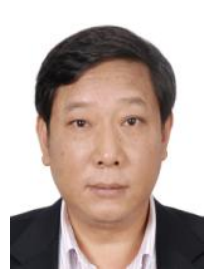

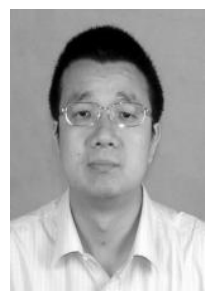

Wenhua Liu (M'03) was born in Hunan, China in 1968. He received his B.E.E. degree, M.E.E and Ph.D. degrees from Tsinghua University, Beijing, China, al in Electrical Engineering, in 1988, 1993 and 1996 respectively. $\mathrm{He}$ is currently a professor in the Department of Electrical Engineering at Tsinghua University, Beijing, China. His main research interests are in high power electronic and Flexible AC Transmission System. 